\title{
Acknowledgement to Reviewers of Infrastructures in 2019
}

Infrastructures Editorial Office

MDPI, St. Alban-Anlage 66, 4052 Basel, Switzerland

Published: 20 January 2020

The editorial team greatly appreciates the reviewers who have dedicated their considerable time and expertise to the journal's rigorous editorial process over the past 12 months, regardless of whether the papers are finally published or not. In 2019, a total of 78 papers were published in the journal, with a median time to first decision of 19 days and a median time from submission to publication of 43 days. The editors would like to express their sincere gratitude to the following reviewers for their generous contribution in 2019:

Abdul Kudus, Sakhiah

Ahmadi, Farzad

Aikawa, Akira

Al Khalifa, Fay

Alfonso Piña, William $\mathrm{H}$.

Alonso Tristán, Cristina

Al-Zwainy, Faiq

Amini, Kamran

Anajafi, Hamidreza

Arabi, Saeed

Arabzadeh, Ali

Arafa, Ahmed

Atadero, Rebecca

Aurangzeb, Qazi

Azimi, Mohsen

Balsa, José

Banica, Alexandru

Bazzaz, Mohammad

Beben, Damian

Bedon, Chiara

Behnood, Ali

Beitelmal, Wesam H.

Binici, Baris

Bobylev, Nikolai

Bocci, Edoardo

Boone, Shane

Botti, Lucia

Bousdar Ahmed, Dina

Cabaleiro, Manuel

Campisi, Tiziana

Cantisani, Giuseppe

Casas, Irene

Castillo Devoto, Gabriel
Chen, Ke

Chorzepa, Mi G.

Congedo, Paolo Maria

Corigliano, Pasqualino

Corradi, Marco

Corrado, Rindone

Couto, António

Cunningham, Keith

Czemplik, Andrzej

D'Amato, Michele

D'Andrea, Antonio

Daszczuk, Wiktor B.

de Brito, Jorge

Delcea, Camelia

Dell'Isola, Marco

Deluka-Tibjaš, Aleksandra

Dhulipala, Somayajulu

Dorafshan, Sattar

Doulos, Lambros T.

Drygała, Izabela Joanna

Dvorský, Tomáš

Easa, Said M.

Edler, Dennis

Eiksund, Gudmund Reidar

Farrokh Ghatte, Hamid

Faruqi, M. A.

Fiorentino, Gabriele

Florentin, Eric

Funari, Marco Francesco

Gago-Calderon, Alfonso

Gallelli, Vincenzo

Gao, Xiaojian

Garcia Cucalon, Lorena 
García-Ros, Gonzalo

Gawronek, Pelagia

Gillen, David

Glas, Andreas Herbert

Glinicki, Michał A

Gomez-Donoso, Francisco

Gonela, Vinay

Goniewicz, Krzysztof

Gopalakrishnan, Kasthurirangan

Granata, Francesco

Hanifehzadeh, Mohammad

Hariri-Ardebili, M. Amin

Henrie, Morgan

Henriques, António

Hernandez-Escobedo, Quetzalcoatl

Hooghoudt, Jan-Otto

Ilbeigi, Mohammad

Imperatore, Stefania

Intini, Paolo

Izadi Moud, Hashem

Jaczewski, Mariusz

Jain, Akhilesh

Janda, Tomas

Jaya Acharya, Nepal

Jhong, Bing-Chen

Jin, Hui

Jin, Qingping

Judd, Johnn P.

Kacprzak, Dominik

Kamiński, Marcin

Kamis, Ahmed Samy

Kang, Sung-Hoon

Kaplan, Sigal

Kara De Maeijer, Patricia

Karimi, Mohammad M.

Kassai, Miklós

Kato, Hironori

Kavroudakis, Dimitris

Keramatikerman, Mahdi

Kermanshachi, Sharareh

Kerres, Karsten

Khan, Bilal

Kim, Jonghyeob

Kim, Sung-Min

Kola, Rashmi

Kong, Xiangxiong

Konstantakopoulos, Ioannis

Kovacevic, M.S.

Kruk, Michael

Kuok, Sin-Chi

Kurpinska, Marzena

Kwoczyńska, Bogusława
Labib, S.M.

Lamperti Tornaghi, Marco

Lantsoght, Eva

Laurini, Eleonora

Lemos, José Vieira

Lester, Henry

Levacher, Daniel

Li, Jun

Li, Yundong

Lim, JongBeom

Lima, Carmine

Ling, Meng

Liu, Jiechao

Lock, Oliver

Loizos, Andreas

Loprencipe, Giuseppe

Lu, Cherie

Lu, Qing

Maazoun, Azer

Mackiewicz, Piotr

Mahoutian, Mehrdad

Malakooti, Amir

Mangalathu, Sujith

Maraveas, Chrysanthos

Marence, Miroslav

Martinelli, Enzo

Mata, Luis Alexander

Mavromatis, Stergios

Mazumder, Mithil

McDaniel, Patrick

Micaelo, Rui

Mikhailenko, Peter

Misa, Rafał

Mityushev, Valdimir

Moini, Mohamadreza

Mojtahedi, Mohammad

Molaei, Maedeh

Moradi, Saeed

Moretti, Laura

Moulos, Vrettos

Nahvi, Ali

Nam, Seong-Nam

Narula, Lakshay

Ngamkhanong, Chayut

Niu, Xirong

Nuss, Larry

Oats, Renee

Obonyo, Esther

Ogierman, Witold

Oh, Taekeun

Ooi, Chinchun

Ostrowski, Krzysztof 
Ouyang, Jian

Pach, Grzegorz

Pakrashi, Vikram

Pastucha, Elzbieta

Patidar, Sandhya

Paultre, Patrick

Polinpapilinho, Katina

Pomoni, Maria

Porras, Rocio

Pourkamali, Farhad

Pratelli, Chiara

Praticò, Filippo Gianmaria

Prieto, Andrés J

Rabiee, Ramin

Radu, Petru Valentin

Radulescu, Virgil Mihai

Rahami, Hossein

Ramezani, Majid

Rassõlkin, Anton

Rau, Jiannyeou

Rizzo, Fabio

Rocha Segundo, Iran

Rodrigues, Eugénio

Rodriguez-Hernandez, Jorge

Roohi, Milad

Rufino, João

Runran, Liu

Rys, Dawid

S. Esmaeeli, Hadi

Sabih, Gauhar

Sallam, Hossam El-Din

Sanavia, Lorenzo

Sanjuán, Miguel Ángel

Santos, Bertha

Santos, Luís Picado

Sasmito, Agus Pulung

Schneider, Jennifer L.

Semendary, Ali A.

Sewalkar, Parag

Sharp, John

Shehu, Rafael

Shi, Xijun

Sierra, Leonardo

Sinha, Nityanand

Siriwardena, Mohan

Sobanjo, John

Sołowczuk, Alicja

Sousa, Luís

Spragg, Robert

Steyn, Wynand JvdM

Strauss, Tim

$\mathrm{Su}$, Jinya
Sun, Congcong

Sun, Jianchun

Susilawati, Susilawati

Suszanowicz, Dariusz

Szmagliński, Jacek

Szymanowski, Jacek

Tafraoui, Ahmed

Tam, Chat-Tim

Tang, Wenzhe

Tang, Xiaochao

Tang, Yunchao

Tatar, Jovan

Tešić, Milan

Tfwala, Samkele

Thives, Liseane

Thives, Liseane Padilha

Thiyagarajan, Jothi Saravanan

Thomas, John E.

Thöns, Sebastian

Thorpe, David

Tokarczyk-Dorociak, Katarzyna

Torbaghan, Mehran Eskandari

Torres, Anthony

Trakas, Dimitris

Truong-Hong, Linh

Tsamatsoulis, Dimitris

Tscheikner-Gratl, Franz

Tutak, Magdalena

Uday, Payuna

Vaiana, Rosolino

Van Wijk, Ian

Vandewalle, Lucie

Vincent, Thomas

Vona, Marco

Vrána, Radek

Wahiduzzaman, Md

Wang, Cao

Wang, Duanyi

wang, shanyong

Wong, Leslie

$\mathrm{Wu}$, John

Xenidis, Yiannis

Xiao, Yue

Xiong, Lin

Xiong, Zhihua

$\mathrm{Xu}$, Jinliang

Yang, Guangwei

Yepes, Víctor

Yi, Junyan

Yu, Huayang

Zambon, Ivan

Zani, Giulio 
Zanni, Mariangela

Zeinali, Yasha

Zhang, Fei
Zhang, Heng

Zhi, Xiaoli

(C) 2020 by the authors. Licensee MDPI, Basel, Switzerland. This article is an open access article distributed under the terms and conditions of the Creative Commons

Attribution (CC BY) license (http://creativecommons.org/licenses/by/4.0/). 